\title{
Sequential BCG and electromotive mitomycin versus BCG alone for high-risk superficial bladder cancer: a randomised controlled trial
}

\begin{abstract}
Savino M Di Stasi, Antonella Giannantoni, Arcangelo Giurioli, Marco Valenti, Germano Zampa, Luigi Storti, Francesco Attisani, Andrea De Carolis, Giovanni Capelli, Giuseppe Vespasiani, Robert L Stephen*
\end{abstract}

\section{Summary}

Background The rationale for combining anticancer drugs has not been applied consistently to use of intravesical agents for treatment of superficial bladder cancer, for which immunotherapeutic BCG and chemotherapeutic mitomycin seem to be a potentially effective combination. We aimed to do a prospective, randomised comparison of BCG alone with that of sequential BCG and electromotive mitomycin in patients with stage pT1 bladder cancer.

Methods After transurethral resection and multiple biopsies, 212 patients with stage pT1 bladder cancer were randomly assigned to: $81 \mathrm{mg}$ BCG infused over 120 min once a week for 6 weeks (n=105); or to $81 \mathrm{mg}$ BCG infused over 120 min once a week for 2 weeks, followed by $40 \mathrm{mg}$ electromotive mitomycin (intravesical electric current $20 \mathrm{~mA}$ for $30 \mathrm{~min}$ ) once a week as one cycle for three cycles ( $\mathrm{n}=107)$. Complete responders underwent maintenance treatment: those assigned BCG alone had one infusion of $81 \mathrm{mg}$ BCG once a month for 10 months, and those assigned BCG and mitomycin had $40 \mathrm{mg}$ electromotive mitomycin once a month for 2 months, followed by $81 \mathrm{mg}$ BCG once a month as one cycle for three cycles. The primary endpoint was disease-free interval; secondary endpoints were time to progression; overall survival; and disease-specific survival. Analyses were done by intention to treat. This trial has been submitted for registration at the US National Cancer Institute website http://clinicaltrials.gov.

Findings Median follow-up was 88 months (IQR 63-110). Patients assigned sequential BCG and electromotive mitomycin had higher disease-free interval than did those assigned BCG alone (69 months [95\% CI 55-86] vs 21 months [15-54]; difference between groups 48 months [42-54], log-rank $\mathrm{p}=0 \cdot 0012$ ). Patients assigned sequential BCG and electromotive mitomycin also had lower recurrence (41.9\% [32.7-51.5] vs 57.9\% [48.7-67.5]; difference between groups $16.0 \%$ [2.7-29.3], log-rank $\mathrm{p}=0.0012)$; progression $(9 \cdot 3 \%$ [3.8-14.8] vs $21.9 \%$ [17.9-25.9]; difference between groups $12.6 \%$ [3.0-22.2], log-rank $\mathrm{p}=0 \cdot 004)$; overall mortality $(21 \cdot 5 \%$ [13.5-29.5] vs $32.4 \%$ [23.4-41.4], difference between groups $10 \cdot 9 \%$ [0.6-21.2], log-rank $\mathrm{p}=0 \cdot 045)$; and disease-specific mortality $(5 \cdot 6 \%$ $[1 \cdot 2-10 \cdot 0]$ vs $16 \cdot 2 \%[6 \cdot 1-23 \cdot 3]$, difference between groups $10 \cdot 6 \%[2 \cdot 5-18 \cdot 7], \log$-rank $\mathrm{p}=0 \cdot 01)$. Side-effects were mainly localised to the bladder.

Interpretation BCG-induced inflammation might increase the permeability of the bladder mucosa such that mitomycin can reach the target tissue more easily and exert its anticancer effect.

\section{Introduction}

Intravesical treatment for superficial bladder cancer has been used for the past $4-5$ decades. Most early reports of such treatment were anecdotal, and the effects were not clarified until the early to mid 1990s. ${ }^{1}$ Intravesical chemotherapy is beneficial in terms of frequency of recurrence and time to recurrence in grade $1-2$ stage Ta tumours, which are usually non-invasive. By contrast, intravesical chemotherapy has negligible effect on disease progression in high-risk superficial bladder cancer-ie, grade 3, stage T1, and carcinoma in situ. However, BCG as induction and maintenance treatment effectively delays progression. ${ }^{2}$

Mitomycin for treatment of bladder cancer has been studied widely, with various doses, concentrations, infusion volumes, and residence times in the bladderusually in non-selected groups of patients. Studies ${ }^{3,4}$ have recorded an incomplete and variable clinical response to intravesical mitomycin, partly as a result of the insensitivity of highly malignant tumours and inadequate drug delivery to tumour cells. Wientjes and colleagues $^{4}$ combined data for laboratory, animal, and human studies with those from computer simulations to derive a mitomycin regimen that would keep diffusion down concentration gradients to an optimum (ie, Fick's first law of diffusion). In a phase III trial, ${ }^{3}$ patients with stage Ta grade 1-2 bladder cancer who were allocated this regimen (40 mg mitomycin, pharmacokinetic manipulation to increase drug concentration by decreasing urine volume, and urine alkalinisation to stabilise the drug) had better time to recurrence and less recurrence than did those with the same stage and grade of cancer allocated standard treatment (20 mg mitomycin). However, data for patients with carcinoma in situ, stage T1, or grade 3 disease were less definitive, although a trend of improved time to recurrence was noted. ${ }^{3}$
Lancet Oncol 2006; 7: 43-51 Published online December 16, 2005 DOI: 10.1016/S1470-2045(05) 70472-1

See Reflection and Reaction page 6

*R L Stephen died in July, 2004 Department of Surgery/Urology, Tor Vergata University, Rome, Italy (Prof S M Di Stasi MD, Prof G Vespasiani MD, F Attisani MD, A De (arolis MD); Department of Urology, University of Perugia, Perugia, Italy (A Giannantoni MD); Operative Unit of Urology, "A Perrino" Hospital, Brindisi, Italy (A Giurioli MD); Section of Medical Statistics and Epidemiology, University of L'Aquila, L'Aquila, Italy (Prof M Valenti MD); Operative Unit of Oncology, S Giacomo Hospital, Rome, Italy (G Zampa MD); Operative Unit of Urology, Policlinico Casilino, Rome, Italy (L Storti MD); Department of Health Sciences, University of Cassino, Cassino

(FR), Italy (Prof G Capelli MD); Physion Laboratories, Medolla (MO), Italy (R L Stephen MD)*

Correspondence to: Prof Savino M Di Stasi, Via Torrice n 4, 00189 Rome, Italy sdistas@tin.it

Fick's first law of diffusion The rate of diffusion or transport of a drug across a biological membrane is directly proportional to the surface area of the membrane and to the concentration gradient, and is inversely proportional to the thickness of the membrane. 
Electromotive mitomycin increases tissue uptake compared with that of passive diffusion. ${ }^{5}$ However, a pilot study ${ }^{6}$ of residual tumour in the bladder after complete transurethral resection in patients with intermediate-risk superficial bladder cancer reported that electromotive mitomycin was no better than passive-diffusion mitomycin (complete response $41.0 \%$ vs $41 \cdot 6 \%$ ). However, patients who responded to electromotive mitomycin had lower recurrence and higher disease-free interval than did patients who responded to passive-diffusion mitomycin. ${ }^{6}$ Furthermore, electromotive mitomycin increases drug delivery by 4-7 times over that of passive transport.? A randomised trial ${ }^{8}$ of 108 patients with multifocal carcinoma in situ (98 of whom had stage T1 tumours removed by transurethral resection) suggested that BCG and electromotive mitomycin were effective treatment for bladder carcinoma in situ, and that when used alone both agents seemed more effective than did passive-diffusion mitomycin. Therefore, electromotive mitomycin has emerged as an alternative or complementary treatment to BCG.

The rationale for combining anticancer drugs, which is now done for most systemic regimens, is based on the need to increase efficacy and reduce emergence of resistant malignant cells. Curiously, this approach is not frequently applied to use of intravesical agents for treatment of superficial bladder cancer, for which immunotherapeutic BCG and chemotherapeutic mitomycin seem to be a potentially effective combination. Studies, ${ }^{9-15}$ mostly from northern Europe, have addressed concurrent use of mitomycin and BCG, and assigned two roles to mitomycin: that of antitumour action and of a tissue-scarifying (ie, surface-modifying) effect that enables BCG to attach more efficiently to the urothelium.

We therefore aimed to assess whether induction of inflammation by use of BCG before mitomycin treatment makes the bladder mucosa more permeable and thus enables mitomycin to reach the target more easily. We did a randomised trial to compare the efficacy of sequential BCG and electromotive mitomycin with that of the current standard of BCG alone for patients with high-risk superficial bladder cancer.

\section{Methods \\ Patients}

All patients with histologically proven stage pT1 transitional-cell carcinoma of the bladder, whether papillary or solid, were regarded as being at high risk for tumour recurrence and at moderate to high risk for progression because of: multifocal pT1, primary or recurrent, grade 2 transitional-cell carcinoma; primary or recurrent pT1, multifocal or solitary, grade 3 transitional-cell carcinoma; or pT1 with carcinoma in situ. Inclusion criteria were: age 18 years or older; adequate bone-marrow reserve (ie, white-blood-cell count $\geqslant 4000 \times 10^{6}$ cells $/ \mathrm{L}$ and platelet count $\geqslant 120 \times 10^{\circ} / \mathrm{L}$ ); normal renal function (ie, serum creatinine $\leqslant 123.76 \mu \mathrm{mol} / \mathrm{L}$ ); normal liver function (ie, serum glutamic-oxaloacetic transaminase $\leqslant 42 \mathrm{U} / \mathrm{L}$, serum glutamic-pyruvic transaminase $\leqslant 48 \mathrm{U} / \mathrm{L}$, and total bilirubin $\leqslant 22.23 \mu \mathrm{mol} / \mathrm{L}$ ); and Karnofsky performance status between 50 and 100 .

Exclusion criteria were: previous treatment with BCG or electromotive mitomycin; treatment with any other intravesical cytostatic agent within the past 6 months; concomitant urothelial tumours of the upper urinary tract; previous muscle-invasive (ie, stage T2 or higher) transitional-cell carcinoma of the bladder; bladder capacity less than $2 \mathrm{~L}$; untreated urinary-tract infection; severe systemic infection (ie, sepsis); urethral strictures that would prevent endoscopic procedures and repeated catheterisation; disease of upper urinary tract (eg, vesicoureteral reflux or urinary-tract stones) that would make multiple transurethral procedures a risk; previous radiotherapy to the pelvis; other concurrent chemotherapy; treatment with radiotherapy-response or biological-response modifiers; history of tuberculosis; other malignant diseases within 5 years of trial registration (except for basal-cell carcinoma); pregnancy or nursing; and psychological, familial, sociological, or geographical factors that would preclude study participation.

\section{Study design}

The institutional review boards of every participating centre approved the study design. Every patient enrolled signed an informed-consent form approved by the institutional review boards.

All patients underwent: urinary cytology of the bladder and upper urinary tract; random cold-cup biopsies of the bladder and prostatic urethra-ie, sampling of seemingly healthy urothelium and of suspicious areas; and complete transurethral resection of all bladder tumour visible on endoscopy, ensuring muscle was included in resected samples. Patients with positive or suspect cytology, carcinoma in situ, multifocal tumours, or grade 3 tumours underwent restaging transurethral resection 4-5 weeks later.

All clinical assessors were adequately trained in the above procedures, and no methods were used to enhance the quality of measurements. All biopsy samples of tumour and bladder were reviewed by a pathologist (AR) for stage and grade. Tumour stage was classified according to the 1997 TNM classification of the International Union Against Cancer, ${ }^{16}$ and tumour grade was defined in accordance with the 1973 WHO classification. ${ }^{17}$

Patients were randomised within 10 days of the first or restaging transurethral resection. Randomisation and data collection were done by use of a central computer. Patients were allocated to BCG alone or to BCG and electromotive mitomycin by use stratified, blocked 
randomisation across 14 (ie, $2^{4}-2$ ) strata as a result of four factors: primary versus recurrent tumours; multifocal versus unifocal tumours; grade 3 versus grade 2 tumours; and presence versus absence of carcinoma in situ. GC generated and coordinated the randomisation process, and obtained data. This study was not blinded because of differences in treatment schedules and drug appearance.

\section{Treatment schedules}

All patients were scheduled to receive their allocated intervention about 3 weeks after transurethral resection and multiple, random biopsy sampling. The BCG instillation consisted of $81 \mathrm{mg}$ wet weight $\left(10 \cdot 2 \pm 9 \cdot 0 \times 10^{8}\right.$ colony-forming units) BCG Connaughtsubstrain (ImmuCyst ${ }^{\circledR}$ Alfa Wassermann SpA, Bologna, Italy). Lyophilised (ie, freeze-dried) BCG was suspended in $50 \mathrm{~mL}$ bacteriostatic-free solution of $0.9 \%$ sodium chloride. After draining of the bladder, the suspension was infused intravesically through a Foley catheter. The solution was retained in the bladder for $120 \mathrm{~min}$, followed by emptying of the bladder and removal of the catheter.

Patients randomly allocated to BCG and mitomycin, were placed on fluid restriction and $2 \mathrm{~g}$ ingested sodium bicarbonate the night before treatment, the morning of treatment, and $2 \mathrm{~h}$ before treatment with mitomycin. ${ }^{16} \mathrm{~A}$ Foley catheter was inserted and, under ultrasonography, postvoid residual volume was reduced to less than $10 \mathrm{~mL} .{ }^{16} 40 \mathrm{mg}$ mitomycin (Mitomycin, Kyowa Italiana Farmaceutici, Srl, Milan, Italy) dissolved in $100 \mathrm{~mL}$ water was infused intravesically through the Foley catheter by gravity and retained in the bladder for $30 \mathrm{~min}$, while $40-60 \mathrm{~mA}$ per s to a maximum of $20 \mathrm{~mA}$ for $30 \mathrm{~min}$ pulsed electric current was given externally. ${ }^{13}$ Two dispersive cathode electrodes were placed on lower abdominal skin that had been degreased with alcohol and a $2-5-\mathrm{mm}$ layer of conductive gel applied. The bladder was then emptied and the catheter removed.

The BCG-alone group was assigned one course of intravesical treatment per week for 6 weeks; patients assigned sequential BCG and electromotive mitomycin were assigned one course of treatment per week for 9 weeks (figure 1), for whom one cycle consisted of two BCG infusions and one mitomycin infusion (three cycles in total, figure 1).

Patients in the BCG-alone group who were diseasefree 3 months after treatment were scheduled to receive monthly infusions of BCG for 10 months (figure 1). Patients in the BCG-and-mitomycin group who were disease-free 3 months after treatment were scheduled to receive one infusion a month for 9 months: three cycles of mitomycin, mitomycin, and BCG (ie, six infusions of mitomycin and three infusions of BCG (figure 1). Maintenance treatment for both groups was given to the same dose and methods of infusion as initial allocated treatment.

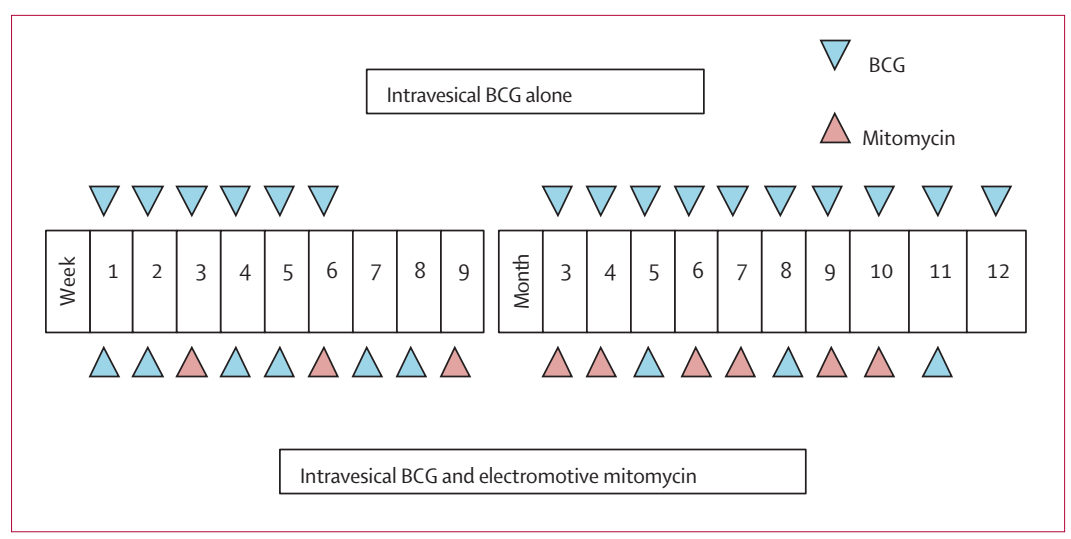

Figure 1: Dose schedules

Response to treatment was assessed with abdominal ultrasonography, cystoscopy, and urinary cytology. In patients who were free of disease 3 months after treatment, these assessments were done every 3 months during the first 3 years and every 6 months thereafter. Patients with carcinoma in situ underwent abdominal ultrasonography, cystoscopy, urinary cytology, and

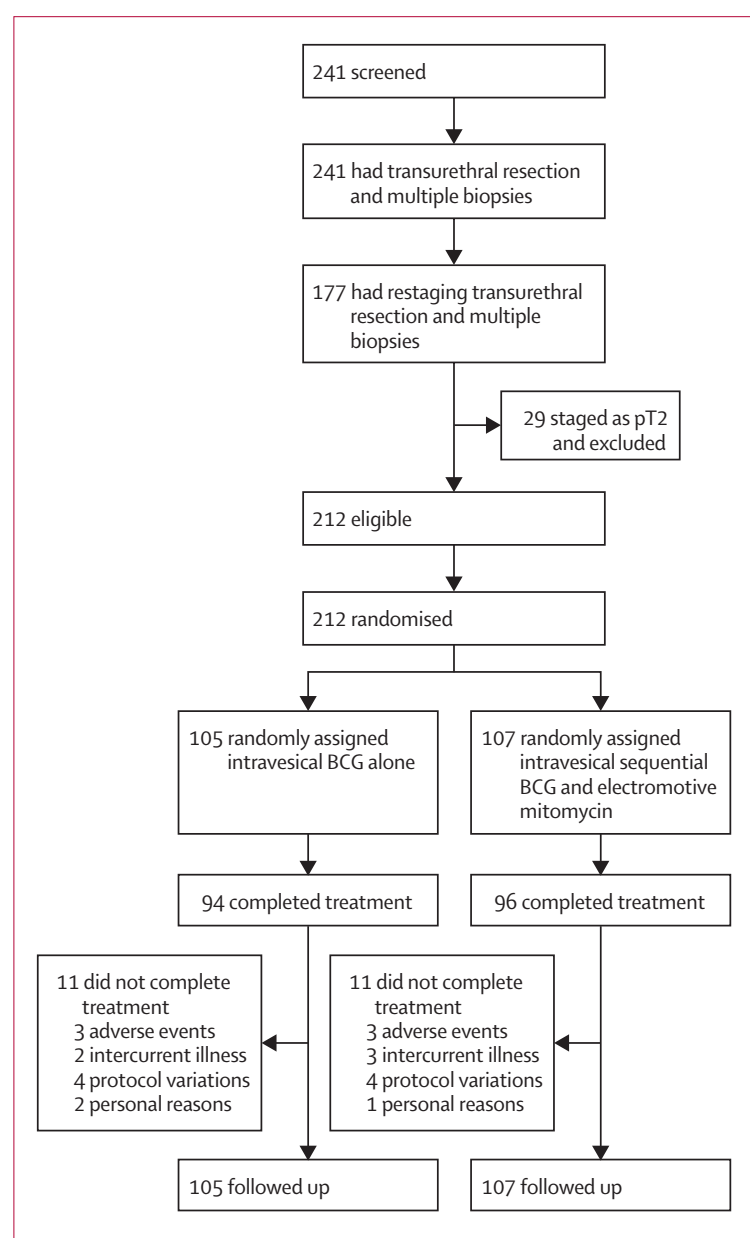

Figure 2: Trial profile 


\begin{tabular}{|lll|}
\hline & $\begin{array}{l}\text { BCG alone } \\
(\mathbf{n}=105)\end{array}$ & $\begin{array}{l}\text { BCG and mitomycin } \\
(\mathbf{n}=107)\end{array}$ \\
\hline Sex & & \\
Men & 86 & 87 \\
Women & 19 & 20 \\
Age (years) & & \\
Median (IQR) & $67(61-73)$ & $66(56-73)$ \\
Follow-up (months) & & \\
Median (IQR) & $84(61-111)$ & $91(65-108)$ \\
Previous intravesical treatment & & \\
Epirubicin & 21 & 25 \\
Mitomycin & 16 & 14 \\
Interferon & 2 & 2 \\
Gemcitabine & 4 & 5 \\
Disease characteristics & & \\
pT1-G2 (all multifocal) & 64 & 65 \\
Primary & 34 & 33 \\
Recurrent & 30 & 32 \\
Concomitant carcinoma in situ & 4 & 4 \\
pT1-G3 & 41 & 42 \\
Primary & 28 & 28 \\
Recurrent & 13 & 14 \\
Unifocal & 20 & 20 \\
Multifocal & 21 & 22 \\
Concomitant carcinoma in situ & 24 & \\
\hline Table 1: Baseline characteristics by treatment group & \\
& & \\
\hline
\end{tabular}

random bladder biopsies at 3 months and 6 months. If bladder cytology was positive for cancer cells but no lesions were visible on cystoscopy, cytology of the upper urinary tract and random biopsies of the bladder and prostatic urethra were done.

If, at 3 months' follow-up, carcinoma in situ persisted or a superficial tumour recurred (ie, stage pTa tumour confined to the urothelium or stage pT1 with invasion of the lamina propria), the patient underwent multiple, random biopsy sampling and transurethral resection, and received a second course of intervention treatment.

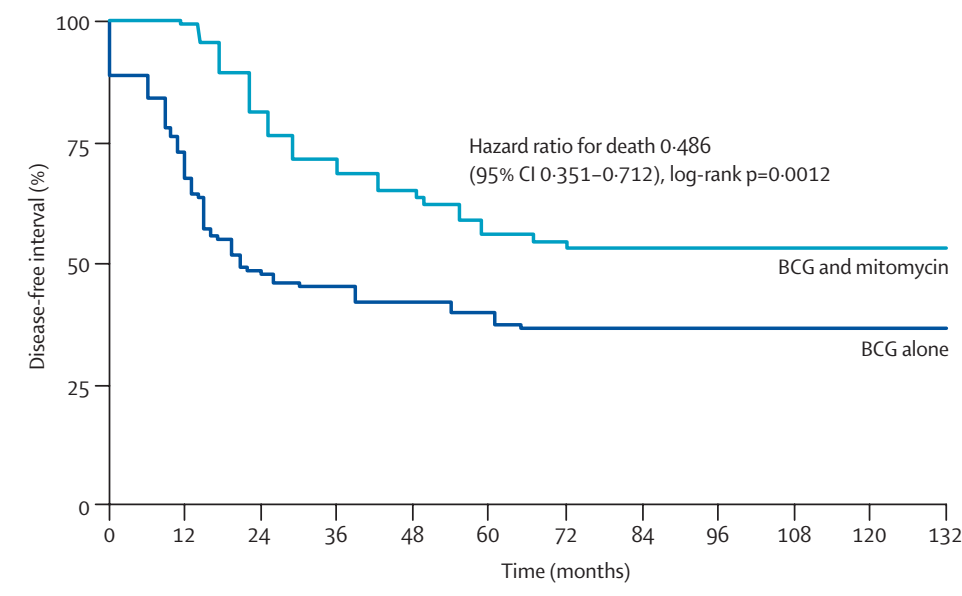

Numbers at risk

$\begin{array}{lllllllllllll}\text { BCG alone } & 105 & 65 & 45 & 41 & 37 & 32 & 26 & 17 & 14 & 9 & 5 & 0\end{array}$

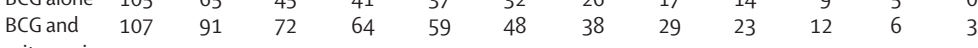

mitomycin
Cystoscopy, biopsies, and urinary cytology were repeated 3 months after the start of the second course. Patients who were disease-free after the second course of treatment received the full course of monthly maintenance instillations (ie, one infusion of BCG for 10 months for those assigned BCG alone, and nine months of sequential BCG and electromotive mitomycin for those assigned combination treatment; figure 1).

After randomisation, patients were suspended from the trial on a second recurrence, on persistence of carcinoma in situ, on development of carcinoma in the upper-urinary tract or prostatic urethra, on progression to muscle-invasive disease (ie, stage pT2 or more advanced), or on development of metastases. Further treatment was left to the discretion of the local investigator.

\section{Toxic effects}

Side-effects were classified as local, systemic, or allergic. Local toxic effects were defined as culture-proven bacterial cystitis, drug-induced (ie, chemical) cystitis, macroscopic haematuria, dysuria, prostatitis, epididymitis, obstruction of the ureter, and contracted bladder; systemic as fever of more than $38^{\circ} \mathrm{C}$, general malaise and fatigue, liver toxicity, BCG-induced lung infection, and BCG sepsis; and allergic as skin rash, arthralgia, and arthritis. The severity of side-effects was classified by the attending physician as those that needed "no delay", "delay", or "indefinite postponement of instillations".

\section{Efficacy endpoints}

Patients with stage pT1 tumour without carcinoma in situ were classified as disease-free and were therefore treated prophylactically; those with carcinoma in situ were treated therapeutically, and response was scored as no response or as complete response. Complete response was defined as complete disappearance of carcinoma in situ, as documented by a normal cytology, cystoscopy, and random bladder biopsies.

The primary endpoint was disease-free interval for patients without carcinoma in situ and for patients with carcinoma in situ who were disease-free after treatment-ie, time from randomisation to first cystoscopy noting recurrence. Patients with carcinoma in situ who did not have complete response after 3 months of treatment were regarded as having recurrence with no follow-up. The secondary endpoints were time to progression, overall survival, and diseasespecific survival. Time to progression was defined as time from randomisation until the onset of muscleinvasive disease as recorded by pathological assessment of transurethral-resection samples or biopsy samples. Overall survival was defined as time from randomisation until death from any cause; diseasespecific survival as time from randomisation until death from bladder cancer. Patients without recurrence or 


\begin{tabular}{|c|c|c|c|c|}
\hline & BCG alone* $(n=105)$ & BCG and mitomycin* $(n=107)$ & Hazard ratio $(95 \% \mathrm{Cl}) \dagger$ & Log-rank $p$ \\
\hline \multicolumn{5}{|l|}{ Recurrence $(n=212)$} \\
\hline pT1-G2 including pTis $(n=129)$ & $33 / 64(51 \cdot 6 \%)$ & $24 / 65(36 \cdot 9 \%)$ & $0.465(0.312-0.841)$ & 0.0221 \\
\hline pT1-G2 excluding $\mathrm{pT}$ is $(\mathrm{n}=121)$ & $30 / 60(50.0 \%)$ & $24 / 61(39 \cdot 3 \%)$ & $0.633(0.370-1.083)$ & 0.0896 \\
\hline pT1-G3 including pTis $(n=83)$ & $28 / 41(68 \cdot 3 \%)$ & $21 / 42(50 \cdot 0 \%)$ & $0.304(0.098-0.643)$ & 0.0165 \\
\hline pT1-G3 excluding pTis $(n=34)$ & $11 / 17(64 \cdot 7 \%)$ & $7 / 17(41 \cdot 2 \%)$ & $0.385(0.148-0.997)$ & 0.0495 \\
\hline pTis $(n=57)$ & $20 / 28(71 \cdot 4 \%)$ & $14 / 29(48 \cdot 3 \%)$ & $0.478(0.200-0.766)$ & 0.0274 \\
\hline \multicolumn{5}{|c|}{ Median disease-free interval $(n=212) \ddagger$} \\
\hline pT1-G2 including $\mathrm{pT}$ is $(\mathrm{n}=129)$ & $45(18-60)$ & $66(58-71)$ & .. & .. \\
\hline pT1-G2 excluding pTis $(n=121)$ & $45(21-60)$ & $66(58-71)$ &.. & .. \\
\hline pT1-G3 including $\mathrm{pT}$ is $(\mathrm{n}=83)$ & $11(9-22)$ & $53(33-68)$ & .. & .. \\
\hline pT1-G3 excluding pTis $(n=34)$ & $14(10-61)$ & NA & .. & .. \\
\hline pTis $(n=57)$ & $9(0-21)$ & $52(27-63)$ & .. & .. \\
\hline \multicolumn{5}{|l|}{ Progression $(n=212)$} \\
\hline pT1-G2 including pTis $(n=129)$ & $6 / 64(9 \cdot 4 \%)$ & $3 / 65(4 \cdot 6 \%)$ & $0.611(0.168-2.112)$ & $0 \cdot 2701$ \\
\hline pT1-G2 excluding $\mathrm{pT}$ is $(\mathrm{n}=121)$ & $5 / 60(8 \cdot 3 \%)$ & $3 / 61(4.9 \%)$ & $0.579(0.138-2.425)$ & 0.4505 \\
\hline pT1-G3 including $\mathrm{pTis}(\mathrm{n}=83)$ & $17 / 41(41 \cdot 5 \%)$ & $7 / 42(16 \cdot 7 \%)$ & $0.400(0.061-0.755)$ & 0.0019 \\
\hline pT1-G3 excluding pTis $(n=34)$ & $1 / 17(5 \cdot 9 \%)$ & $2 / 17(11 \cdot 8 \%)$ & $1 \cdot 320(0 \cdot 11-15 \cdot 87)$ & 0.8200 \\
\hline pTis $(n=57)$ & $17 / 28(60 \cdot 7 \%)$ & $5 / 29(17 \cdot 2 \%)$ & $0.171(0.062-0.467)$ & $<0.0001$ \\
\hline \multicolumn{5}{|l|}{ Death from any causes $(n=212)$} \\
\hline pT1-G2 including $\mathrm{pT}$ is $(\mathrm{n}=129)$ & $12 / 64(18 \cdot 8 \%)$ & $13 / 65(20 \cdot 0 \%)$ & $0.930(0.601-1.482)$ & 0.9008 \\
\hline pT1-G2 excluding $\mathrm{pT}$ is $(\mathrm{n}=121)$ & $11 / 60(18 \cdot 3 \%)$ & $13 / 61(21 \cdot 3 \%)$ & $0.930(0.621-1.392)$ & 0.7012 \\
\hline pT1-G3 including $p$ Tis $(n=83)$ & $22 / 41(53 \cdot 7 \%)$ & $10 / 42(23 \cdot 8 \%)$ & $0.511(0.105-0.826)$ & 0.0009 \\
\hline pT1-G3 excluding $\mathrm{pTis}(\mathrm{n}=34)$ & $5 / 17(29 \cdot 4 \%)$ & $2 / 17(11 \cdot 8 \%)$ & $0.901(0.402-2.022)$ & 0.0685 \\
\hline pTis $(n=57)$ & $18 / 28(64 \cdot 3 \%)$ & $8 / 29(27 \cdot 6 \%)$ & $0.288(0.106-0.777)$ & 0.0026 \\
\hline \multicolumn{5}{|l|}{ Death from bladder cancer $(n=212)$} \\
\hline pT1-G2 including pTis $(n=129)$ & $2 / 64(3 \cdot 1 \%)$ & $1 / 65(1 \cdot 5 \%)$ & $0.512(0.041-5.529)$ & 0.5500 \\
\hline pT1-G2 excluding pTis $(n=121)$ & $2 / 60(3 \cdot 3 \%)$ & $1 / 61(1.6 \%)$ & $0.501(0.045-5.526)$ & 0.5606 \\
\hline pT1-G3 including $p$ Tis $(n=83)$ & $15 / 41(36 \cdot 6 \%)$ & $5 / 42(11.9 \%)$ & $0.227(0.091-0.420)$ & 0.0031 \\
\hline pT1-G3 excluding pTis $(n=34)$ & $1 / 17(5 \cdot 9 \%)$ & $1 / 17(5 \cdot 9 \%)$ & $0.467(0.023-9.618)$ & 0.6109 \\
\hline pTis $(n=57)$ & $14 / 28(50 \cdot 0 \%)$ & $4 / 29(13.8 \%)$ & $0.206(0.067-0.631)$ & 0.0022 \\
\hline \multicolumn{5}{|c|}{$\begin{array}{l}\text { PTis=carcinoma in situ. NA=not available: median disease-free interval estimates time after which } 50 \% \text { of study group are expected to survive. For diseases with favourable prognosis, } \\
\text { survival probability might not reach } 0.5 \text { during study period (as was the case for time to progression and survival in our study), preventing estimate of median time or upper } 95 \% \mathrm{Cl} \text {. } \\
\text { *Data are number (\%), unless otherwise indicated. TReference risk of time-to-event for BCG-alone group 1.0. } \neq \text { Data are months ( } 95 \% \mathrm{Cl} \text { ); data for hazard ratio and log-rank p as those for } \\
\text { recurrence. }\end{array}$} \\
\hline
\end{tabular}

progression were censored at the last cystoscopy, and those lost to follow-up were censored at the last known day of survival.

\section{Statistical methods}

All analyses were done by intention to treat. The primary endpoint was used to determine the sample size. Previously reported data ${ }^{18,19}$ and our own experience formed the basis of an assumption that the probability of being recurrence-free at 5 years was about $60 \%$ in patients with high-risk superficial bladder cancer treated with BCG, and that an increase of $20 \%$ in time to recurrence was clinically relevant. Thus, for a type I error of $\alpha=0.05$ and power $1-\beta=0 \cdot 80$, the required sample size is 173 . Allowance for potential withdrawal of $15 \%$ increased the sample size to about 210-ie, 105 patients per treatment group. A total of 52 events (ie, recurrence) was required.

Time to first recurrence, time to progression, overall survival, and disease-specific survival were estimated by use of the Kaplan-Meier method. Comparisons were estimated by use of log-rank test. All tests were twosided, and $\mathrm{p}<0 \cdot 05$ was regarded as significant.

We calculated hazard ratios with $95 \%$ CI by use of proportional-hazards regression.

\section{Role of the funding source}

The sponsor of the study had no role in study design; in the collection, analysis, or interpretation of data; or in

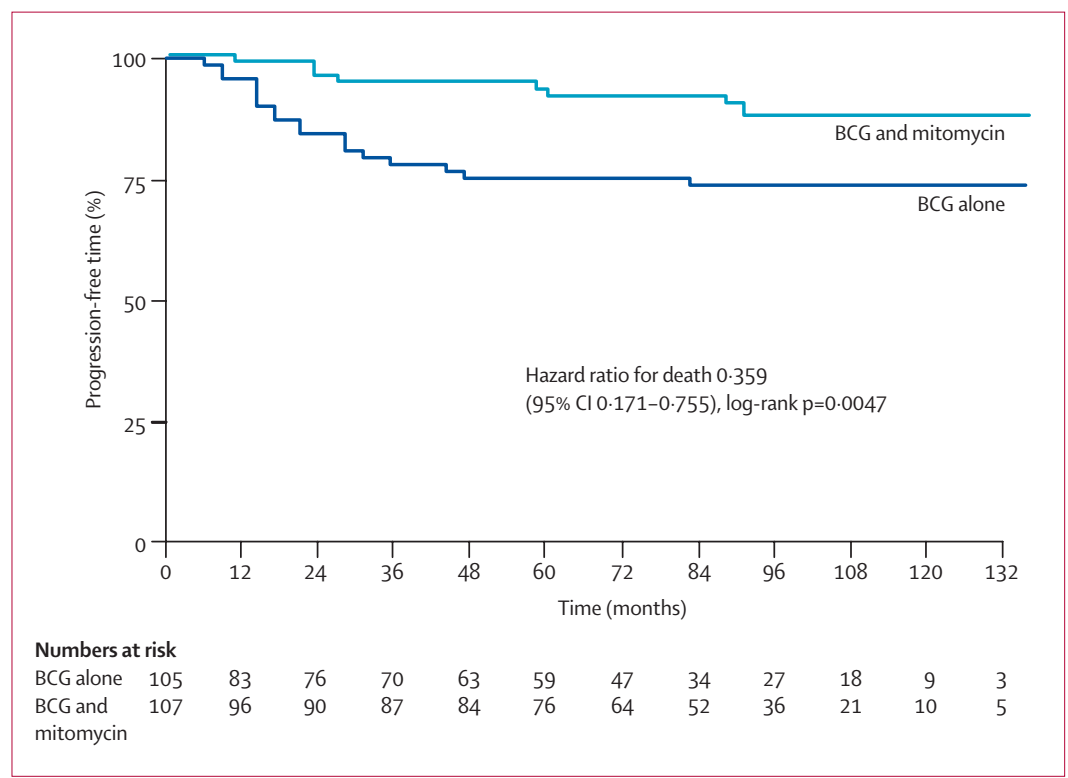

Figure 4: Progression-free time 


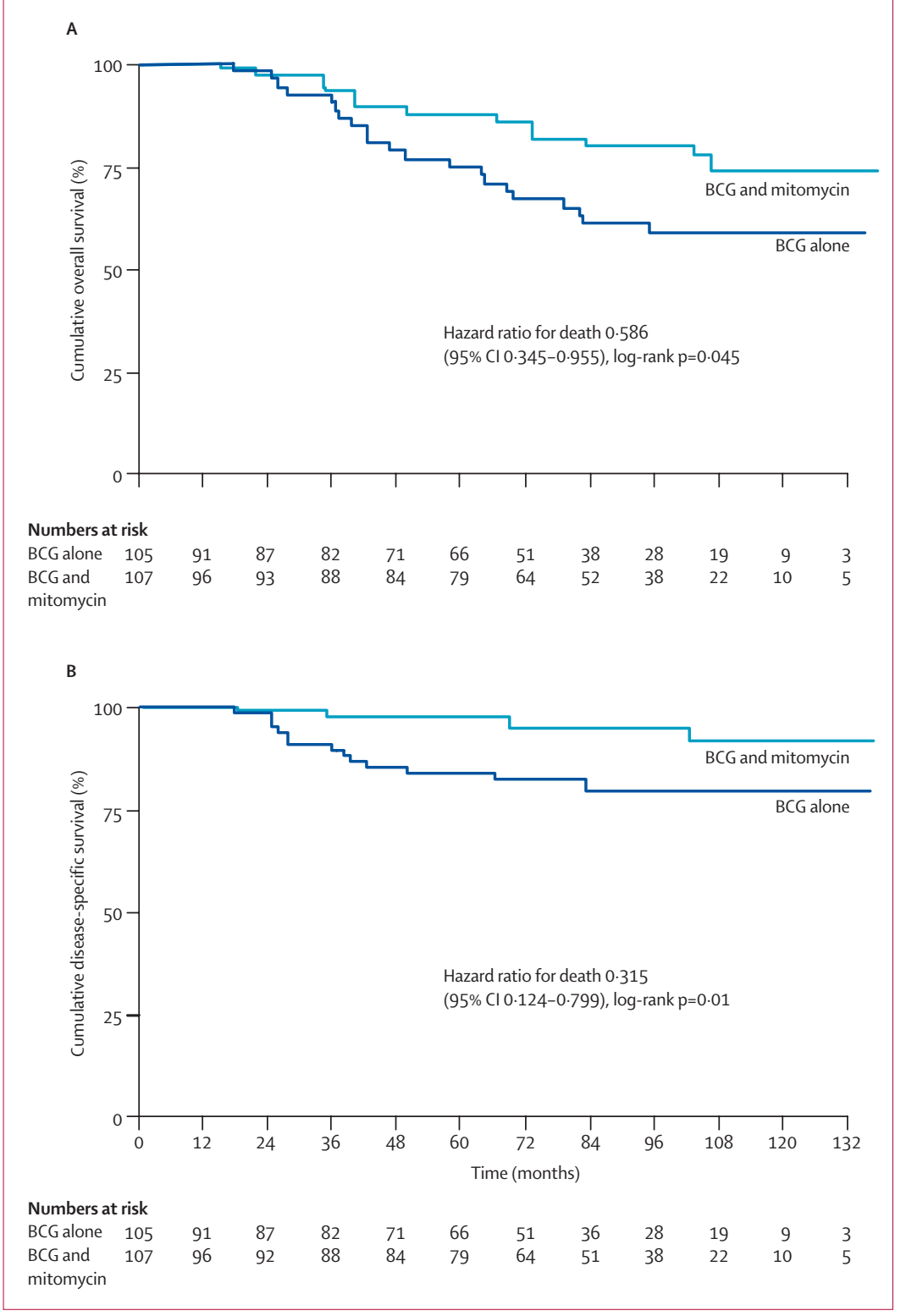

Figure 5: Overall survival and disease-specific survival

(A) Overall survival. (B) Disease-specific survival.

the writing of the report. The corresponding author had full access to all the data in the study and had final responsibility for the decision to submit for publication.

\section{Results}

Between Jan 1, 1994 and June 30, 2002, 241 patients were enrolled at four different centres (figure 2). On restaging transurethral resection, 56 of 177 patients had residual stage pTa, pT1 tumour or carcinoma in situ (superficial) on histological examination and were therefore eligible. Figure 2 shows the trial profile; table 1 shows baseline characteristics by treatment group. Because the actual number of events was 86 , the power of the trial was $95 \%$.

\begin{tabular}{|lll|}
\hline & $\begin{array}{l}\text { BCG alone } \\
(\mathbf{n = 1 0 5 )}\end{array}$ & $\begin{array}{l}\text { BCG and mitomycin } \\
(\mathbf{n}=\mathbf{1 0 7 )}\end{array}$ \\
\hline Event & 51 & 54 \\
Dysuria & 14 & 16 \\
Bacterial cystitis & 46 & 49 \\
Drug-induced cystitis & 61 & 64 \\
Macroscopic haematuria & 1 & 0 \\
Prostatitis & 0 & 1 \\
Epididymitis & 24 & 21 \\
Fever & 34 & 33 \\
Influenza-like symptoms & 32 & 32 \\
Fatigue & & 64 \\
Treatment suspended & 65 & 40 \\
No & 37 & 3 \\
Yes, temporarily (up to 5 days) & 3 & \\
Yes, indefinitely & \\
\hline Table 3: Adverse effects in patients who received at least one or more \\
intravesical treatment
\end{tabular}

All 78 patients with stage pT1 disease without carcinoma in situ who were assigned sequential BCG and electromotive mitomycin (table 1 ) were disease-free after the first course of treatment: all started maintenance treatment at 3 months. 72 of 77 patients with stage pT1 disease without carcinoma in situ who were allocated BCG alone were disease-free after the first course of treatment, and started maintenance treatment; the remaining five patients in this treatment group, who were not disease-free, received a second course of BCG, to which all responded and were given maintenance treatment at 6 months.

After a median follow-up of 88 months (IQR 63-110), 106 patients were disease-free, including $44(41.9 \%)$ in the BCG-alone group and $62(57.9 \%)$ in the BCG-andmitomycin group $(\mathrm{p}=0 \cdot 0012)$. Thus, patients assigned sequential BCG and electromotive mitomycin had higher disease-free interval than did those assigned BCG alone (69 months [95\% CI 55-86] vs 21 months [15-54]; difference between groups 48 months [42-54], log-rank $\mathrm{p}=0 \cdot 0012$; figure 3 ). Table 2 shows disease-free interval and recurrence by treatment group according to stage of disease.

Figure 4 shows time to progression by treatment group. Patients assigned sequential BCG and electromotive mitomycin had lower progression than did those assigned BCG alone $(9 \cdot 3 \%$ [3.8-14.8] vs $21.9 \%$ [17.9-25.9]; difference between groups $12 \cdot 6 \%$ [3·0-22·2], log-rank p=0·0047; table 2). 33 (16\%) of 212 patients progressed to muscle-invasive disease: 23 in BCG-alone group and ten in the BCG-and-mitomycin group. 20 of 33 patients (15 assigned BCG alone and five assigned BCG and mitomycin) with biopsy-confirmed muscle-invasive bladder cancer underwent cystectomy, and 13 (eight assigned BCG alone and five assigned BCG and mitomycin) who refused, or were unfit for, radical surgery underwent transurethral resection and chemotherapy plus radiation. After randomisation, five of ten patients in the BCG-and-mitomycin group 
progressed between 14 months and 33 months, and five of ten between 42 months and 91 months; 20 of 23 patients allocated BCG alone progressed between 6 months and 35 months, and three between 44 months and 83 months.

Patients assigned sequential BCG and electromotive mitomycin had a better outlook than did those assigned BCG alone. During follow-up, 57 (27\%) of 212 patients died from any cause: 34 in the BCG-alone group and 23 in the BCG-and-mitomycin group (table 2). 23 deaths were due to bladder cancer (17 in the BCG-alone group and six in the BCG-and-mitomycin group, table 2). Figure 5 shows overall survival and disease-specific survival by treatment group. Patients assigned sequential BCG and electromotive mitomycin had lower overall mortality than did those assigned BCG alone $(23$ [21.5\%] of $107[13 \cdot 5-29 \cdot 5]$ vs 34 [32.4\%] of 105 [23.4-41.4], difference between groups $10.9 \%$ $[0 \cdot 6-21 \cdot 2]$, log-rank $\mathrm{p}=0 \cdot 045)$, and disease-specific mortality $(5 \cdot 6 \% \quad[1 \cdot 2-10 \cdot 0]$ vs $16 \cdot 2 \% \quad[6 \cdot 1-23 \cdot 3]$, difference between groups $10 \cdot 6 \%[2 \cdot 5-18 \cdot 7]$, log-rank $\mathrm{p}=0 \cdot 01$ ). Groups did not differ in the frequency or severity of side-effects (table 3 ). Three patients in each group had to withdraw from the study because of severe side-effects.

Although this trial was not designed to assess the response of patients with carcinoma in situ, we compared complete response between groups. At 3 months, $12(42.9 \%)$ of 28 patients with carcinoma in situ assigned BCG alone responded to treatment (95\% CI 24.5-62.8), compared with $16(55 \cdot 2 \%)$ of 29 patients $(35 \cdot 7-73 \cdot 5)$ assigned BCG and mitomycin (difference between groups $12 \cdot 3 \%[-1 \cdot 5$ to $26 \cdot 1], p=0 \cdot 431)$. At 6 months, $16(57 \cdot 1 \%)$ of 28 patients assigned BCG alone responded to treatment $(37 \cdot 2-75 \cdot 5)$, compared with 20 $(69.0 \%)$ of 29 assigned BCG and mitomycin $(49 \cdot 2-84 \cdot 7)$ (difference between groups $11 \cdot 9 \%[-0 \cdot 9$ to 24.7]; $\mathrm{p}=0 \cdot 417)$.

\section{Discussion}

We have shown that intravesical, sequential BCG and electromotive mitomycin for patients with high-risk superficial bladder leads to higher disease-free interval, lower recurrence and progression, and to improved overall survival and disease-specific survival compared with BCG alone. We have also shown that these findings were maintained for some subgroups of patients stratified by tumour stage and grade. To the best of our knowledge, this is the first time simultaneous reduction of all of the above endpoints has been noted. Furthermore, we noted no significant differences in toxic effects between groups.

In patients with carcinoma in situ, complete response after treatment with sequential BCG and electromotive mitomycin tended to be higher than after BCG alonetreatment that is regarded as the most effective intravesical agent for high-risk superficial bladder cancer. $^{20}$ On application of sequential BCG and electromotive mitomycin for the first time, the frequency and severity of side-effects was unknown. In a previous study, ${ }^{8}$ we noted that: intravesical electromotive delivery increased mitomycin transport; plasma concentrations of mitomycin remained adequately below toxic threshold; and that electromotive mitomycin was associated with fewer side-effects than was BCG. Although it is important not to underestimate the toxic effects of intravesical, sequential BCG and electromotive mitomycin, close follow-up and regular communication with patients is thought to keep safety to a maximum. Moreover, we have shown in this trial that overall toxic effects associated with sequential BCG and electromotive mitomycin are no worse than those associated with BCG alone.

In this trial, BCG-induced inflammation might have made the bladder mucosa more permeable so that mitomycin reached the target tissue more easily. We chose the ratio of BCG to mitomycin as two to one to keep toxic effects to a minimum and to enhance efficacy. However, we do not know whether the therapeutic effects of mitomycin were additive or synergistic with those of BCG during initial treatment. We propose the following events based on the timing of infusions: after two BCG treatments, the immune response is established, and tumour cells are exposed to an infiltration of cytokines (some of which exert an antiproliferative action), cytotoxic $\mathrm{T}$ lymphocytes, helper $\mathrm{T}$ lymphocytes, and to several more specific and nonspecific BCG-induced responses..$^{21}$ These cascades seem to be a pivotal moment for the introduction of mitomycin, electromotive delivery of which substantially increases the rate of mitomycin transport and therefore its concentration in tissue. ${ }^{7}$ After enzymatic reduction, ${ }^{22}$ metabolites of mitomycin add to the anticancer effects by cross-linking of DNA to all tissue layers of the bladder wall that are affected by superficial bladder cancer (ie, the urothelium and lamina propria). ${ }^{7}$ Whether cytotoxic lymphocytes are more susceptible to the anticancer effects of mitomycin than are cancer cells remains to be determined. Our findings suggest that mitomycin attacks BCG-resistant cancer cells. However, BCG exerts a direct antiproliferative effect on cell lines of human urothelial carcinoma, which does not seem to be a consequence of apoptosis, but rather of cell-cycle arrest at entry into $\mathrm{S}$ phase after $\mathrm{G} 1$ phase as a result of crosslink-initiated signal transduction to regulatory genes of the cell cycle. ${ }^{23}$ However, mitomycin in bladder tissue could inhibit or destroy viable BCG infused 1 week later, but is unlikely with a total-body clearance of about $18 \mathrm{~L} \mathrm{~h}^{-1} \mathrm{~m}^{-2} \cdot{ }^{24}$

In our trial, the scheduling of mitomycin and the electromotive delivery of mitomycin might account for discrepancies with results of other investigators who have assessed combination mitomycin and BCG. The Finnbladder group, ${ }^{9-13}$ who gave mitomycin before BCG 
in patients with superficial bladder cancer, reported that the timing of infusions ensured that the vesicant effect of mitomycin had mostly subsided on BCG infusion; however, an animal study ${ }^{25}$ reported no evidence that mitomycin potentiated the immune response to BCG. The Finnbladder group ${ }^{9-13}$ and Witjes and co-workers ${ }^{14}$ gave mitomycin by passive diffusion, and the encouraging results achieved by the Finnbladder group with mitomycin and BCG in patients with carcinoma in situ ${ }^{9}$ might have been due to the separate action of BCG. A randomised trial ${ }^{8}$ of 108 patients with multifocal carcinoma in situ suggested that BCG and electromotive mitomycin were both effective as treatment for bladder carcinoma in situ and that both seemed more effective than did mitomycin by passive diffusion. Therefore, the timing schedule and delivery techniques used in our trial were selected to keep the benefits of both drugs at an optimum. Interestingly, a report ${ }^{26}$ showed that percutaneous sequential BCG and mitomycin given as a ratio of BCG to mitomycin as two to one effectively cleared synchronous panurothelial carcinomatosis, with long-term disease free survival.

The optimum maintenance regimen of BCG remains to be defined, and our monthly infusions for those assigned BCG alone is one of several recommendations. ${ }^{2}$ The maintenance schedule in our trial for those assigned sequential BCG and electromotive mitomycin (in a ratio of BCG to mitomycin of one to two) is much the same as that used by Rintala and colleagues.' Because the immune response to BCG fades after about 6 months, ${ }^{20}$ a booster dose every 3 months is reasonable. Electromotive delivery probably exploits the potential of mitomycin to eliminate malignant cells that escape immunological surveillance induced by BCG. ${ }^{8}$

The number of patients $(73 \%)$ who underwent restaging transurethral resection compares favourably with that reported by $\operatorname{Herr}^{27}$ (215 [62\%] of 347). A substantial proportion of patients with high-risk stage T1 bladder cancer have residual disease after initial transurethral resection, and the goals of restaging transurethral resection are to detect residual tumour, obtain disease-free resection, and to reduce the tumour burden before adjuvant treatment. ${ }^{28}$ Furthermore restaging transurethral resection ensures better risk stratification, and for patients with high-risk superficial bladder cancer this technique is mandatory if bladder preservation is intended. ${ }^{29}$

If you would like to respond to an article published in The Lancet Oncology, please email your submission to the Editor at david.collingridge@lancet.com
With 10-year hindsight, what changes in the management of patients with high-risk superficial bladder cancer would be beneficial? First, restaging transurethral resection must be done for all patients with stage T1 bladder cancer: 29 understaged patients identified after meticulous initial transurethral resection was surprising. Second, perioperative mitomycin infusions reduce intraoperative seeding of malignant cells, ${ }^{12,30}$ and should be used in initial and restaging transurethral resection. Third, the optimum dose and schedule of intravesical BCG need to be established because the most commonly used induction and maintenance regimens are arbitrary. Immunotherapeutic BCG is not associated with a smooth dose-response curve, and dose can be reduced to $54 \mathrm{mg}$ or even to $27 \mathrm{mg}$, which maintains therapeutic efficacy while reducing sideeffects. ${ }^{31}$ Finally, the role and mechanism of action of BCG and electromotive mitomycin as maintenance treatment remain to be clarified.

\section{Contributors}

S M Di Stasi was the principal investigator, and provided primary conception, design, logistics, data analysis, and preparation of the paper; A Giannantoni and A Giurioli were the co-principal investigators, and had a role in design, logistics, data analysis, and paper preparation; $G$ Vespasiani coordinated the study and contributed to writing of the paper; G Zampa, L Storti, F Attisani, and A De Carolis recruited and treated patients, and contributed to writing of the paper; G Capelli coordinated the randomisation process and data collection; M Valenti managed statistical analyses, and contributed to editing of the paper; and R L Stephen was the senior team member, and contributed to the primary conception and design of the study.

\section{Conflict of interest}

We declare no conflicts of interest.

\section{Acknowledgments}

Robert L Stephen was a scientific consultant at Physion Srl (Mirandola, MO, Italy), and died on July 1, 2004, in Boston, MA, USA, after 16 months of hospital stay. We thank Antonio Ricci (Pathology Department, Policlinico Sandro Pertini, Rome, Italy) for his collaboration and Geraldine Anne Boyd (University of Perugia, Italy) for help in editing. The study was supported by state grants to "Tor Vergata" University of Rome, Rome, Italy.

References

1 Smith JA, Labasky RF, Cockett ATK, et al. Bladder cancer clinical guidelines panel summary report on the management of nonmuscle invasive bladder cancer (stages Ta, T1 and Tis). J Urol 1999; 162: 1697-701.

2 Sylvester RJ, van der Meijden APM, Lamm DL. Intravesical bacillus Calmette-Guèrin reduces the risk of progression in patients with superficial bladder cancer: a meta-analysis of the published results of randomized clinical trials. J Urol 2002; 168: 1964-70.

$3 \mathrm{Au} \mathrm{JL}$, Badalament RA, Wientjes MG, et al. Methods to improve efficacy of intravesical mitomycin C: results of a randomized phase III trial. J Natl Cancer Inst 2001; 93: 597-604.

4 Wientjes MG, Badalament RA, Au JL. Use of pharmacologic data and computer simulations to design an efficacy trial of intravesical mitomycin $\mathrm{C}$ treatment for superficial bladder cancer. Cancer Chemother Pharmacol 1993; 32: 255-62.

5 Di Stasi SM, Vespasiani G, Giannantoni A, et al. Electromotive delivery of mitomycinc $\mathrm{C}$ into human bladder wall. Cancer Res 1997; 57: 875-80.

6 Brausi M, Campo B, Pizzocaro G, et al. Intravesical electromotive administration of drugs for treatment of superficial bladder cancer: a comparative phase II study. Urology 1998; 51: 506-09.

7 Di Stasi SM, Giannantoni A, Massoud R, et al. Electromotive versus passive diffusion of mitomycin $\mathrm{C}$ into human bladder wall: concentration-depth profiles studies. Cancer Res 1999; 59: 4912-18.

8 Di Stasi SM, Giannantoni A, Stephen RL, et al. Intravesical electromotive mitomycin $\mathrm{C}$ versus passive transport mitomycin $\mathrm{C}$ for high risk superficial bladder cancer: a prospective randomized study. J Urol 2003; 170: 777-82.

9 Rintala E, Jauhiainen K, Rajala P, et al. Alternating mitomycin C and bacillus Calmette-Guèrin instillation treatment for carcinoma in situ of the bladder. J Urol 1995; 154: 2050-53.

10 Rintala E, Jauhiainen K, Kaasinen E, et al, for the Finnbladder Group. Alternating mitomycin C and bacillus Calmette-Guèrin instillation prophylaxis for recurrent papillary (stages Ta to T1) superficial bladder cancer. J Urol 1996; 156: 56-59. 
11 Kaasinen E, Rintala E, Pere AK, et al. Weekly mitomycin C followed by monthly bacillus Calmette-Guerin or alternating monthly interferon-alpha2B and bacillus Calmette-Guerin for prophylaxis of recurrent papillary superficial bladder carcinoma. J Urol 2000; 164: 47-52.

12 Kaasinen E, Rintala E, Hellstrom P, et al. Factors explaining recurrence in patients undergoing chemoimmunotreatment regimens for frequently recurring superficial bladder carcinoma. Eur Urol 2002; 42: 167-74.

13 Kaasinen E, Wijkstrom H, Malmstrom PU, et al. Alternating mitomycin C and BCG instillations versus BCG alone in treatment of carcinoma in situ of the urinary bladder: a nordic study. Eur Urol 2003; 43: 637-45.

14 Witjes JA, Caris CTM, Mungan NA, et al. Results of a randomized phase III trial of sequential intravesical treatment with mitomycin $\mathrm{C}$ and bacillus Calmette-Guèrin versus mitomycin $\mathrm{C}$ alone in patients with superficial bladder cancer. J Urol 1998; 160: 1668-71.

15 Van der Meijden APM, Hall RR, Macaluso MP, Pawinsky A, et al. Marker tumour response to the sequential combination of intravesical treatment with mitomycin-C and BCG-RIVM in multiple superficial bladder tumours. Eur Urol 1996; 29: 199-203.

16 Sobin LH, Wittekind CH, UICC (International Union Against Cancer). TNM classification of malignant tumours, 5th edn. New York: Wiley Liss, 1997: 107-90.

17 Mostofi FK, Sobin LH, Torloniu H. Histological typing of urinary bladder tumours: International Classification of Tumours, No 10. Geneva: World Health Organization, 1973.

18 Lamm DL, Blumenstein BA, Crawford ED, et al. A randomized trial of intravesical doxorubicin and immunotreatment with bacille Calmette-Guerin for transitional-cell carcinoma of the bladder. N Engl J Med 1991; 325: 1205-09.

19 Melekos MD, Chionis HS, Paranychianakis GS, Dauaher HH. Intravesical 4'-epi-doxorubicin (epirubicin) versus bacillus Calmette-Guerin. A controlled prospective study on the prophylaxis of superficial bladder cancer. Cancer 1993; 72: 1749-55.

20 Kim JC, Steinberg GD. The limits of bacillus Calmette-Guèrin for carcinoma in situ of the bladder. J Urol 2001; 165: 745-56.
21 Alexandroff AB, Jackson AM, O’Donnell MA, James K. BCG immunotreatment of bladder cancer: 20 years on. Lancet 1999; 353: 1689-94.

22 Gan Y, Mo Y, Kalns JE, et al. Expression of DT-Diaphorase and Cytochrome P450 Reductase correlates with mitomycin C activity in human bladder tumours. Clin Cancer Res 2001; 7: 1313-19.

23 Chen F, Zhang G, Iwamoto Y, See WA. BCG directly induces cell cycle arrest in human transitional carcinoma cell lines as consequence of integrin cross-linking. BMC Urol 2005; 5: 8.

24 Den Hartigh J, Mc Vie JG, van Oort WJ, Pinedo HM. Pharmacokinetics of mitomycin C in humans. Cancer Res 1983; 43: 5017-21.

25 Balemans LT, Vegt PD, Steerenberg PA, et al. Effects of sequentia intravesical administration of mitomycin $\mathrm{C}$ and bacillus CalmetteGuèrin on the immune response in the guinea pig bladder. Urol Res 1994; 22: 239-45.

26 Di Stasi SM, Giannantoni A, Stephen RL, et al. Percutaneous sequential bacillus Calmette-Guerin and mitomycin C for panurothelial carcinomatosis. Can J Urol 2005; 12: 2895-98.

27 Herr HW. Restaging transurethral resection of high risk superficial bladder cancer improves the initial response to bacillus CalmetteGuerin treatment. J Urol 2005; 174: 2134-37.

28 Soloway MS, Sofer M, Vaidya A. Contemporary management of stage T1 transitional cell carcinoma of the bladder. J Urol 2002; 167: 1573-83.

29 Grimm MO, Ackermann R. Transurethral resection of superficial bladder cancer: technically safe, oncologically anything but perfect. J Urol 2005; 174: 2086-87.

30 Solsona E, Iborra I, Ricos JV, et al. Effectiveness of a single immediate mitomycin $\mathrm{C}$ instillation in patients with low risk superficial bladder cancer: short and long-term follow up. J Urol 1999; 161: 1120-23.

31 Martinez-Pineiro JA, Martinez-Pineiro L, Solsona E, et al. Has a 3-fold decreased dose of bacillus Calmette-Guerin the same efficacy against recurrences and progression of T1G3 and Tis bladder tumours than the standard dose? Results of a prospective randomized trial. J Urol 2005; 174: 1242-47. 